\title{
Tensile Deformation and Recrystallization in Copper Single Crystal and Bicrystal with Schmid Factor of 0.5
}

\author{
Tatsuya Okada, Hiroshi Kinoshita*1, Minoru Tagami and Fukuji Inoko*2 \\ Department of Mechanical Engineering, Faculty of Engineering, The University of Tokushima, Tokushima 770-8506, Japan
}

In order to study the kind band (KB) formation and its effect on recrystallization in copper, single crystals and an asymmetric bicrystal in which the primary slip system had a Schmid factor of 0.5 , were deformed in tension and subsequently annealed. Several indents were made on the surface of one of the single crystals to promote KB formation. In kink bands (KBs), slip systems on the critical slip plane were activated, which induced the rotation of KBs in the direction opposite to that of the deformation matrix (DM). The orientation difference between the DM and $\mathrm{KB}$ reached $20^{\circ}$ to $25^{\circ}$ at a nominal tensile strain of 0.4 . The KBs in the bicrystal were about ten times broader than those in the single crystals. After annealing, recrystallized grains having twin-relationships one another formed in the bicrystal and the single crystal with indents. A comparison was made with aluminum single crystals and bicrystals of the same orientation. [doi:10.2320/matertrans.MBW200808]

(Received October 17, 2008; Accepted November 26, 2008; Published January 21, 2009)

Keywords: copper, single crystal, bicrystal, tensile deformation, recrystallization, kink band

\section{Introduction}

It is generally accepted that recrystallization preferentially occurs in non-uniformly deformed regions, such as in deformation bands and in the vicinity of grain boundaries. ${ }^{1)}$ In order to analyze the slip geometry and the variation in crystallographic orientation in and around non-uniformly deformed regions, experiments using orientation-controlled single crystals and bicrystals are beneficial. ${ }^{2-10)}$

Deformation bands are classified into kink bands (KBs), ${ }^{11)}$ bands of secondary slip and special types of bands of secondary slip. ${ }^{12)}$ Well-developed KBs are often observed in tensile-deformed aluminum single crystals in which the primary slip system has a Schmid factor of $0.5 .^{13)}$ The activation of the primary slip system in the deformation matrix (DM) rotates the crystal so that the primary slip direction approaches the tensile axis. When the nominal tensile strain exceeds 0.1 , the critical slip system is activated in KBs. The rotation of KBs is opposite to that of the DM. A detailed analysis of slip bands reveals that localized compressive deformation occurs in KBs. ${ }^{13)}$ After annealing, recrystallized grains (RGs) form at the boundaries between the DM and KBs. The crystallographic orientation of each recrystallized grain $(\mathrm{RG})$ is related to that of the DM or the kink band $(\mathrm{KB})$ by its rotation about one of $\langle 111\rangle$ axes. In other words, RGs have $\langle 111\rangle$-rotation relationships with the $\mathrm{DM}$ or the KBs.

There are two types of bicrystals composed of grains with a Schmid factor of 0.5 . The first type (Type I) is a symmetric bicrystal, in which the crystallographic orientations of the component grains are symmetric about the grain boundary. The second type (Type II) is an asymmetric bicrystal, in which the primary slip systems of the component grains are symmetrically arranged about the grain boundary but the crystallographic orientations are not. The tensile deformation and recrystallization of both types of bicrystals were studied

\footnotetext{
${ }^{* 1}$ Graduate Student, The University of Tokushima. Present address Mitsubishi Electric Co. Ltd., Tokyo 100-8310, Japan

${ }^{* 2}$ Emeritus Professor, The University of Tokushima
}

for aluminum. ${ }^{14,15)}$ In Type I bicrystals, ${ }^{14)}$ the slip deformation as well as the arrangement of KBs was almost symmetric about the grain boundary. After annealing, recrystallization occurred through strain-induced boundary migration (SIBM) mechanisms, i.e., each component grain migrated into the adjacent grain along the KBs. On the other hand, in Type II bicrystals, ${ }^{15)}$ the appearance of slip deformation was not symmetric, i.e., a double-slip region formed in one grain due to the activation of primary and secondary slip systems in the vicinity of the grain boundary, and KBs formed only in the other grain. After annealing, no large-scale SIBM occurred, but RGs formed along the KBs through $\langle 111\rangle$-rotation mechanisms.

Compared to aluminum, a relatively small number of studies have been carried out concerning the tensile deformation and recrystallization of orientation-controlled single crystals and bicrystals of copper. It is apparent that the slip bands are finer and the KBs are narrower in copper than in aluminum. ${ }^{11,16)}$ This difference is attributable to the lower stacking fault energy of copper. However, no systematic study has been carried out of the relationship between the development of $\mathrm{KBs}$ and recrystallization.

In the present study, copper single crystals and a Type II bicrystal with a Schmid factor of 0.5 were tensile-deformed and annealed. In one of the single crystals, several indents were made on the surface prior to the tensile deformation to examine the effect of stress concentration sites on the $\mathrm{KB}$ formation and recrystallization.

\section{Experimental Procedures}

A copper bicrystal of Type II orientation was grown with the Bridgman method in a vacuum furnace under a pressure lower than $4 \times 10^{-3} \mathrm{~Pa}$. The purity of the material was 99.99 mass\%. The shape of the bicrystal is shown as a schematic in Fig. 1(a). The stereographic projections of the orientation of component grains A and B are presented in Fig. 1(b) and (c), respectively. The triangular and elliptic symbols correspond to the slip plane normal P1 to P4 and the slip direction D1 to D6, respectively. In the following, the 

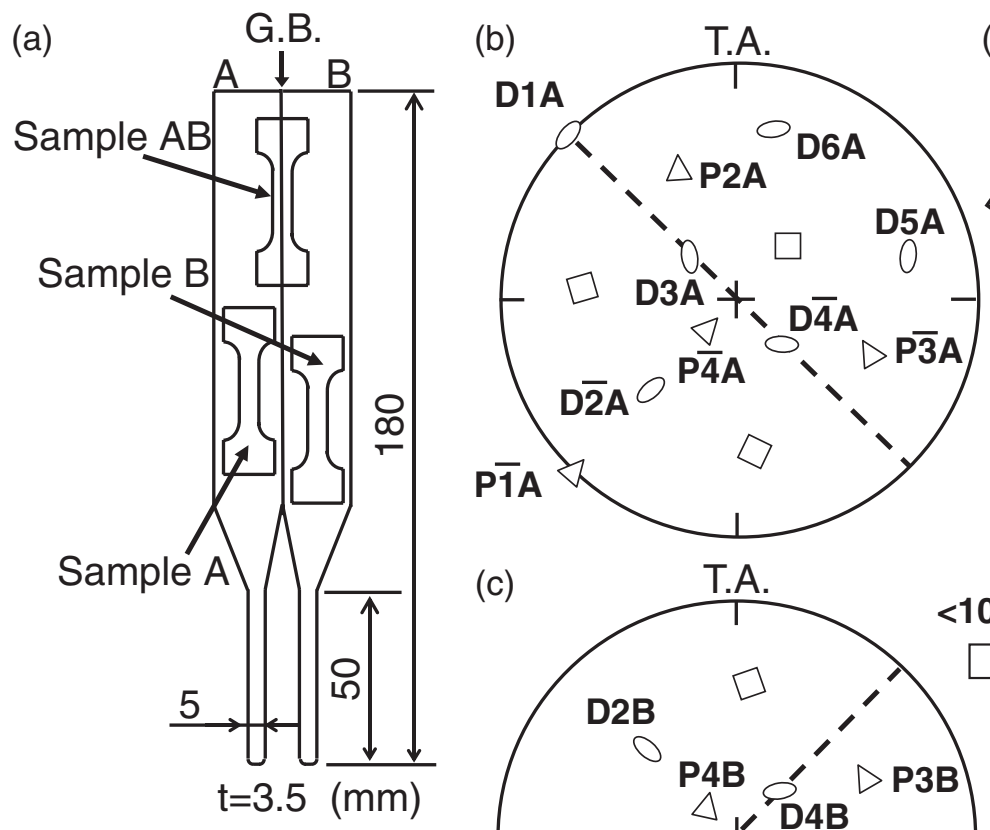

(d)

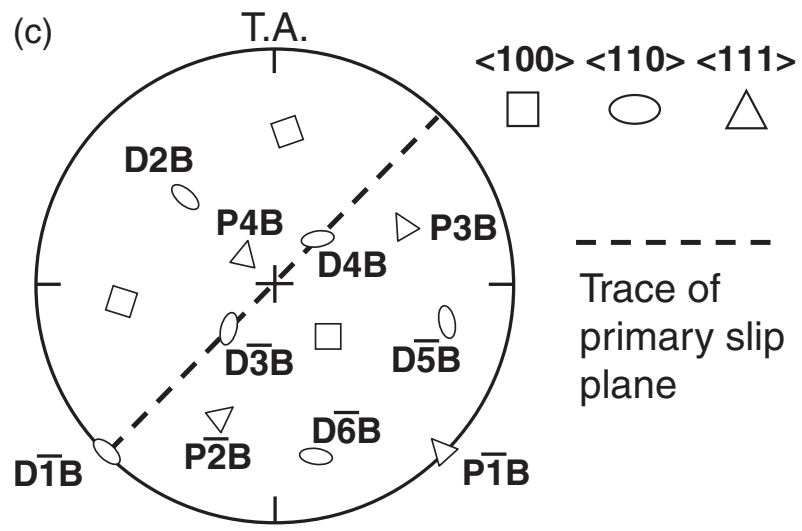

Fig. 1 (a) Shape and size of the bicrystal from which three tensile samples were spark-cut. (b) Initial orientation of Grain A. (c) Initial orientation of Grain B. (d) Shape and size of tensile sample.

slip system is represented by a combination of the slip plane and slip direction along with the name of the grain, i.e., A or $\mathrm{B}$ at the end. P1, P2, P3 and P4 are the primary, critical, conjugate and cross slip planes, respectively, and D1 is the primary slip direction. The direction with a bar on the top of the number is opposite to the one without a bar, i.e., D 1 is the direction opposite to D1. In Fig. 1(b) and (c), the slip traces of the primary slip plane, i.e., P1A and P1B, on the (112) surface are drawn using dashed lines. It is apparent that the primary slip systems, P1D1A and P1D1B, are symmetric about the grain boundary. We should note that the crystallographic orientations of grains are not symmetric but instead are related to each other by a $90^{\circ}$ rotation about the [112] direction, normal to the bicrystal surface. Schmid factors of slip systems calculated from the initial orientation are listed in Table 1.

As shown in Fig. 1(a), three tensile samples were spark-cut from the Type II bicrystal. The shape of the tensile sample is shown as a schematic in Fig. 1(d). The gauge portion of the sample is $4 \times 12 \times 3 \mathrm{~mm}^{3}$. In the following, the bicrystal sample in which the grain boundary is parallel to the tensile axis will be referred to as Sample AB. Two single crystal samples spark-cut from the component grains A and B will be referred to as Sample A and Sample B, respectively. The (112) surfaces of the samples were mechanically polished and subsequently electropolished in a solution of phosphoric acid in ethanol. On the (112) surface of Sample A, six indents with an interval of $1 \mathrm{~mm}$ were made using a Vickers hardness tester.
Table 1 Schmid factors of slip systems

\begin{tabular}{cccc}
\hline Slip plane & Slip direction & Grain A & Grain B \\
\hline \multirow{3}{*}{ P1 } & D1 & 0.49 & 0.50 \\
\cline { 2 - 4 } & D3 & 0.26 & 0.24 \\
\cline { 2 - 4 } & D4 & 0.23 & 0.26 \\
\cline { 2 - 4 } P2 & D2 & 0.48 & 0.46 \\
\cline { 2 - 4 } & D4 & 0.26 & 0.31 \\
\hline \multirow{2}{*}{ P3 } & D5 & 0.20 & 0.16 \\
\cline { 2 - 4 } & D2 & 0.19 & 0.21 \\
\hline \multirow{2}{*}{ P4 } & D3 & 0.12 & 0.13 \\
\cline { 2 - 4 } & D6 & 0.30 & 0.34 \\
\cline { 2 - 4 } & D1 & 0.15 & 0.18 \\
\hline
\end{tabular}

A nominal tensile strain of 0.4 was applied to the samples at room temperature, with an initial strain rate of $3 \times 10^{-4} \mathrm{~s}^{-1}$. The slip pattern and the orientation variation were analyzed with an optical microscope (OM), a scanning electron microscope (SEM), electron channeling pattern (ECP) and electron backscatter diffraction (EBSD).

Three samples were annealed in an air furnace. After annealing, the oxide film on the sample surface was removed by etching with nitric acid. Then, the furnace temperature was raised, and the same processes were repeated until RGs 


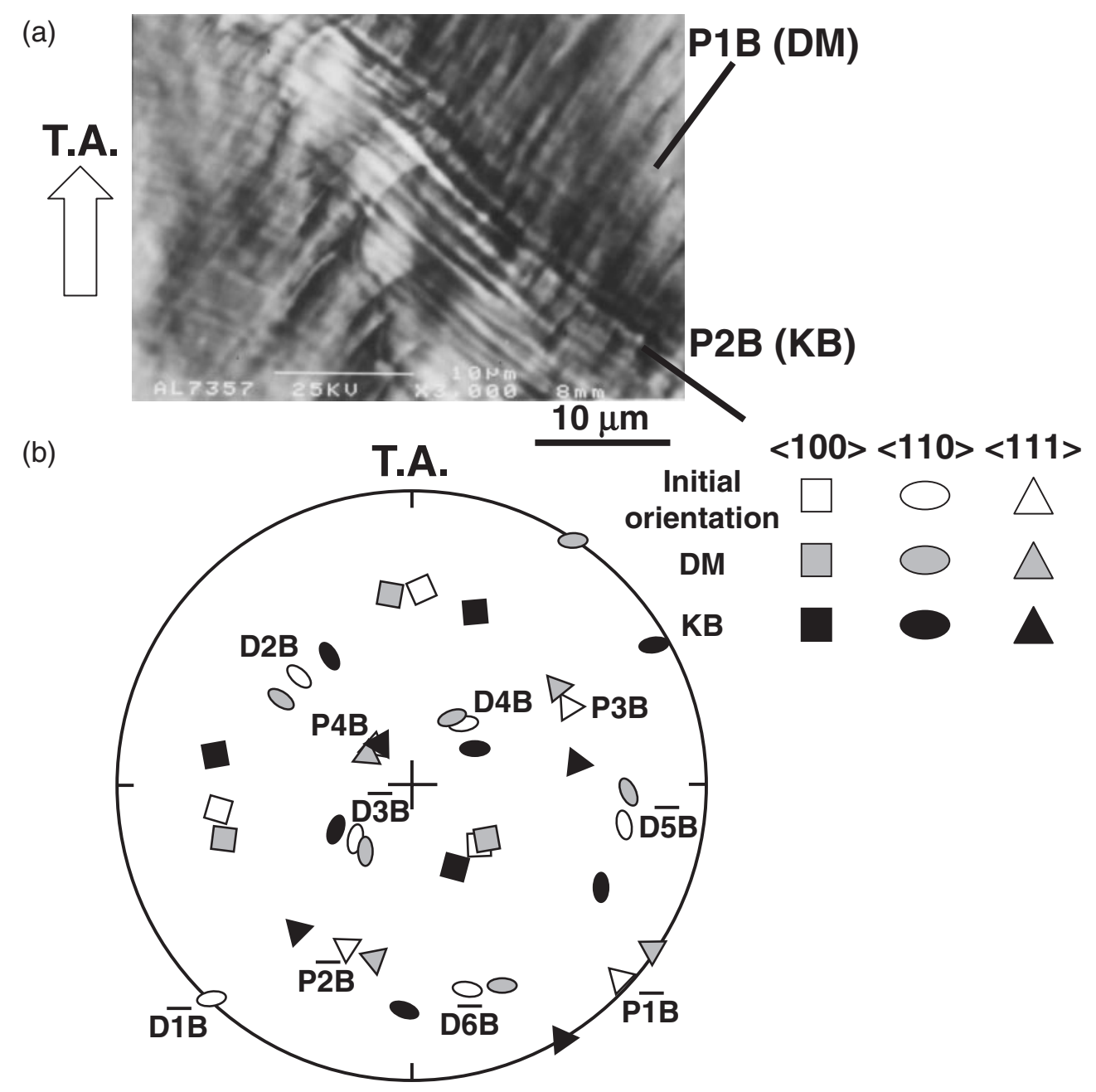

Fig. 2 (a) SEM image of slip bands in a KB in Sample B. (b) Stereographic projection of the initial orientation, and the DM and KB orientations in Sample B.

were found with an OM. The arrangement and the crystallographic orientation of RGs were analyzed with SEM/ECP and SEM/EBSD techniques.

\section{Results and Discussion}

\subsection{Effect of Vickers indents on KB formation}

In Sample B, the activation of the primary slip system P1D1B in the DM rotated the crystal in a counterclockwise (CCW) direction. At a nominal tensile strain of 0.4 , many narrow bands formed, making an angle approximately $65^{\circ}$ to the primary slip. Since the width of these bands was smaller than a few $\mu \mathrm{m}$, a slip pattern within the bands was not observable with SEM. Here, we focused our attention on the bands in which the slip pattern was clearly visible with SEM. In Sample B, there was only one such band, which had a width of approximately $10 \mu \mathrm{m}$. A SEM image of the band is shown in Fig. 2(a). This band has the features of a KB in that a secondary slip system on the critical slip plane P2B is activated and also in that the primary slip bands are bent in the clockwise $(\mathrm{CW})$ direction, opposite to the rotation of the DM.

The rotation associated with this $\mathrm{KB}$ was also confirmed by ECP analyses. The orientations of the DM and $\mathrm{KB}$ are presented in a stereographic projection in Fig. 2(b). The DM and $\mathrm{KB}$ are rotated with respect to the initial orientation about the [112] axis in CCW and CW directions, respectively. The misorientation, i.e., the orientation difference between the DM and $\mathrm{KB}$, is $25^{\circ}$. As shown in Fig. 3, the orientation map constructed from EBSD data clearly visualizes this rotation. The crystallographic orientation projected onto the tensile axis is used to construct this orientation map because the DM and KB, being misoriented about the [112] axis, cannot be distinguished when the orientation that is projected in the [112] direction is used for mapping. In Fig. 3, the points having an EBSD image quality lower than a specific value are presented as dark spots. From their distribution, it is presumed that the dislocations accumulate at the boundaries between the DM and KB so as to lower the image quality.

Sample A shows six KBs having a width of approximately $10 \mu \mathrm{m}$ that were formed from the Vickers indents. The appearance of slip bands around one of the KBs shown in Fig. 4(a) is very similar to that in Fig. 2(a). As shown in Fig. 4(b), the DM and $\mathrm{KB}$ are rotated by $10^{\circ}$ in the $\mathrm{CW}$ and CCW directions, respectively, with respect to the initial orientation. The misorientation between the $\mathrm{DM}$ and $\mathrm{KB}$ is $20^{\circ}$. The similarity of the crystallographic rotation associated 


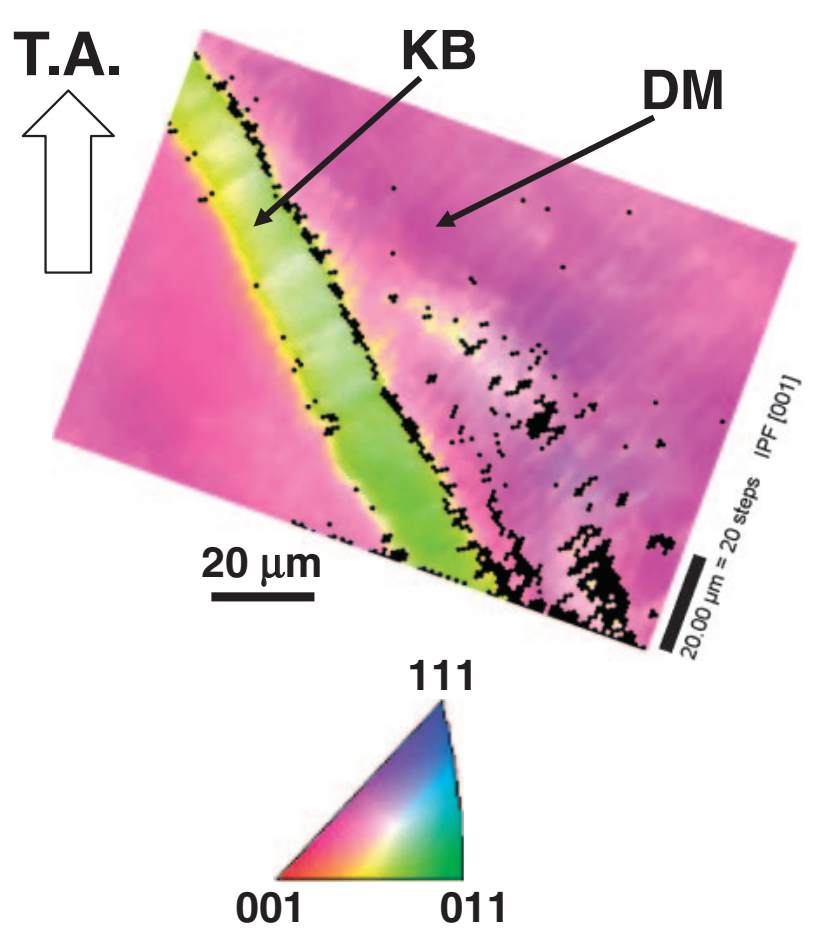

Fig. 3 EBSD orientation map of a KB in Sample B. The color corresponds to the crystallographic orientation projected onto the tensile axis. with the KBs in Sample B and Sample A is apparent from a comparison of the orientation maps in Figs. 3 and 5. The colors of the DM and KB corresponding to the crystallographic orientation projected onto the tensile axis are almost the same. In addition, we also notice in Figs. 3 and 5 a very similar arrangement of dark spots with low image quality at the $\mathrm{DM} / \mathrm{KB}$ boundaries.

In summary, the surface indents induce the formation of KBs but do not affect the width, slip geometry and crystallographic rotation.

\subsection{Effect of grain boundary on KB formation}

In Sample AB, the slip deformation was asymmetric about the grain boundary. An OM image of the gauge portion is shown in Fig. 6(a). KBs are found only in Grain A. The width of KBs in Sample AB is approximately $100 \mu \mathrm{m}$, much larger than that in single crystals. A SEM image of slip bands close to the grain boundary is shown in Fig. 6(b). In the KB in Grain A, slip bands in critical slip plane P2A are predominant. On the other hand, in Grain $\mathrm{B}$, in addition to the primary slip system P1D1B, a secondary slip system on the conjugate slip plane $\mathrm{P} 3 \mathrm{~B}$ is activated to form a doubleslip region in the vicinity of the grain boundary. Among three slip systems on the $\mathrm{P} 3 \mathrm{~B}$ plane, the $\mathrm{P} 3 \mathrm{D} 2 \mathrm{~B}$ system with a Schmid factor of 0.21 has a relatively large stress trans-

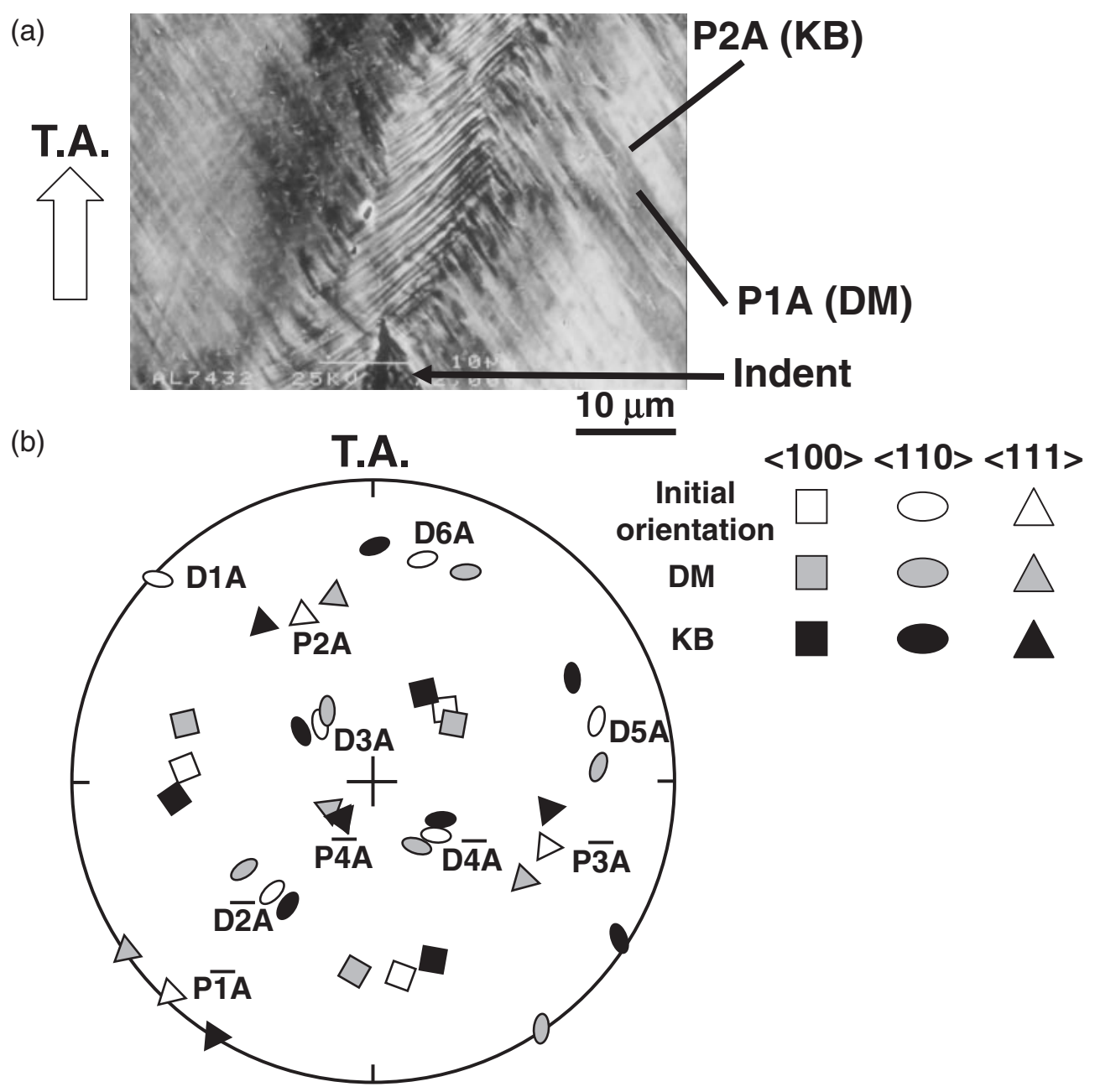

Fig. 4 (a) SEM image of slip bands in a KB from a Vickers indent in Sample A. (b) Stereographic projection of the initial orientation, and the DM and KB orientations in Sample A. 

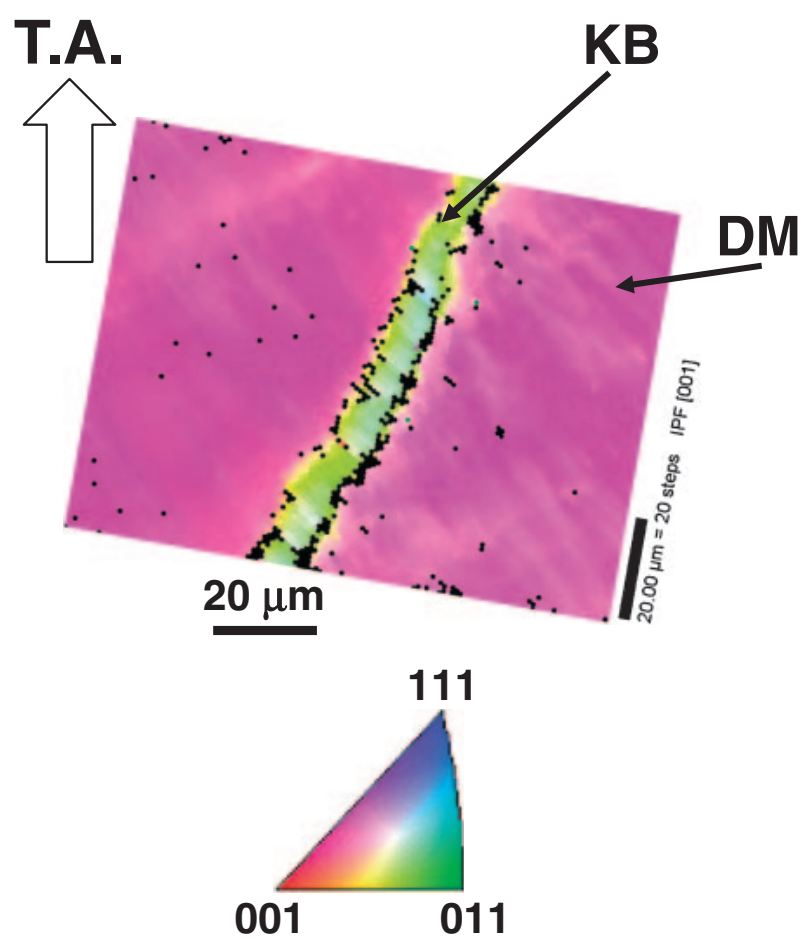

Fig. 5 EBSD orientation map of a KB in Sample A. The color corresponds to the crystallographic orientation projected onto the tensile axis. mission factor ${ }^{17)}$ of 0.68 , with the primary slip system P1D1A in the adjacent Grain A. Presumably, the P3D2B slip system was activated in the initial stage of deformation by the stress transmission from $\mathrm{P} 1 \mathrm{D} 1 \mathrm{~A}$, resulting in the asymmetric deformation of the bicrystal.

The slip geometry of the present copper bicrystal is similar to that in an aluminum bicrystal of Type II orientation in that KBs formed only in one grain and also that a secondary slip system on the conjugate slip plane was activated in the vicinity of the grain boundary in the other grain. ${ }^{15)}$ Crystallographic orientations of the DM and KB in Grain A measured from ECP are presented as a stereographic projection in Fig. 7. The DM and $\mathrm{KB}$ were rotated by $11^{\circ}$ in the $\mathrm{CW}$ and $\mathrm{CCW}$ directions, respectively, with respect to the initial orientation. The misorientation is $22^{\circ}$. Although the width of $\mathrm{KBs}$ in the bicrystal is about ten times larger than that in single crystals, the crystallographic rotation associated with $\mathrm{KBs}$ is similar.

\subsection{Recrystallization}

No RG was found in Sample B even after annealing at $1173 \mathrm{~K}$ for $600 \mathrm{~s}$. Sample A and Sample AB recrystallized after annealing for $180 \mathrm{~s}$ at $973 \mathrm{~K}$ and $823 \mathrm{~K}$, respectively. These results correspond well with the development of KBs in the samples. (a)

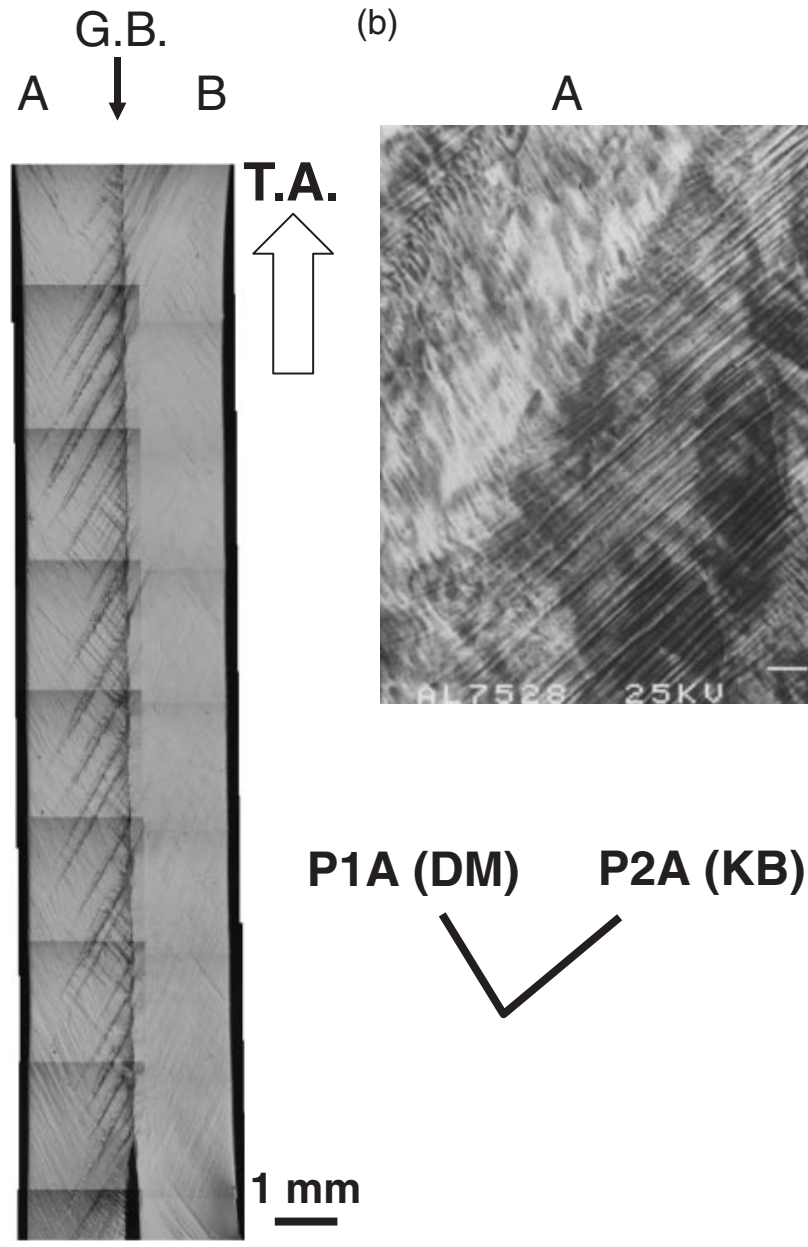

G.B.<smiles>[B]</smiles>

$B$

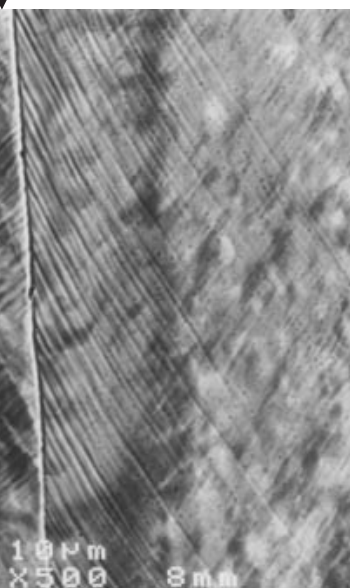

$10 \mu \mathrm{m}$

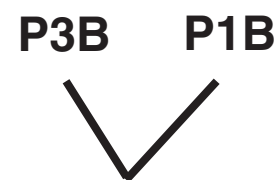

Fig. 6 (a) OM image of Sample AB. (b) SEM image of slip bands close to the grain boundary in Sample AB. 


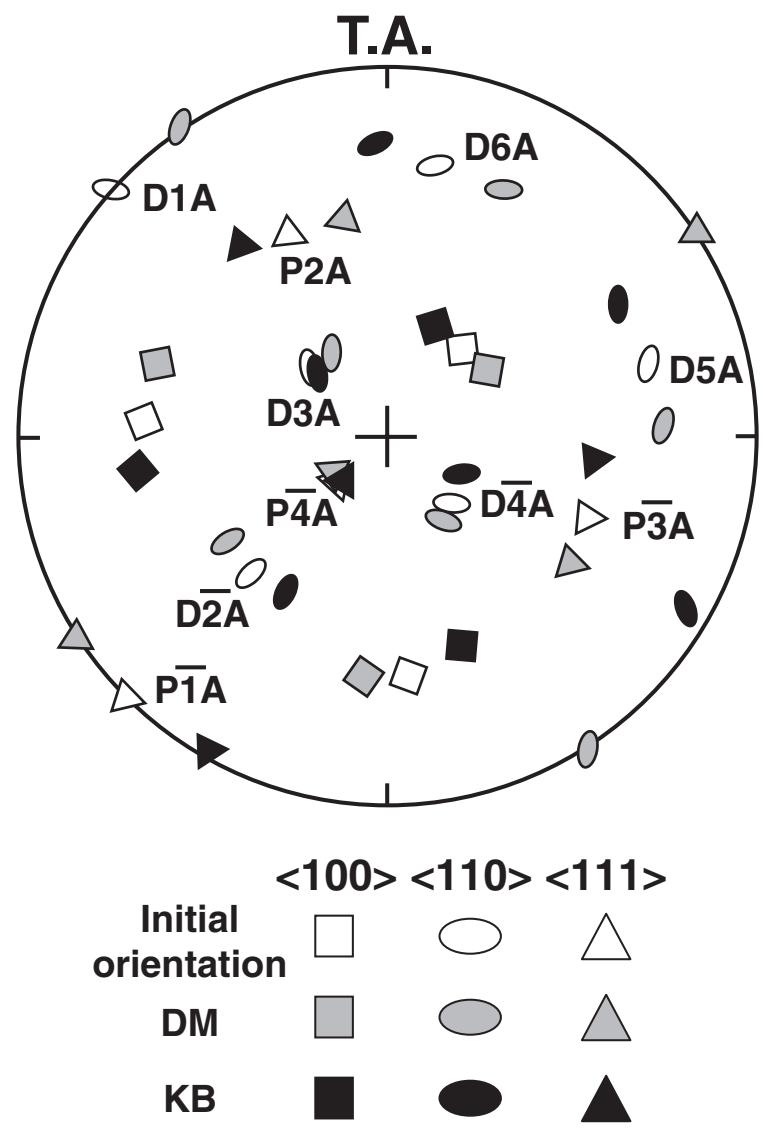

Fig. 7 Stereographic projection of the initial orientation, and the DM and $\mathrm{KB}$ orientations in Grain A of Sample AB.

A SEM image of recrystallized Sample A is shown in Fig. 8(a). Coarse RGs suggest a small number of recrystallization nuclei. Crystallographic orientations of RGs were measured from their electron channeling patterns (ECPs). Twenty-two RGs in the gauge portion are classified into eleven orientations. In Fig. 8(b), RGs are colored according to their orientation. Twin relationships between RGs are shown using arrows in Fig. 8(c).

A SEM image of recrystallized Sample AB is shown in Fig. 9(a). Twenty-three RGs are found in this area. The higher density of RGs suggests a larger number of recrystallization nuclei in Sample AB than in Sample A. EBSD analyses were applied to measure the crystallographic orientation of RGs, as shown in Fig. 9(b). Except for a very small-scale SIBM, e.g., area X in Fig. 9(b), recrystallization occurred through the formation of RGs along the KBs. Twenty-three RGs are classified into six orientations. Twin relationships between the DM and RGs are shown as a schematic in Fig. 10. In the figure, green and dark blue RGs have twin relationships with the DM. Other RGs (pink, red and light blue) are twins of these first-order twins, i.e., green and dark blue RGs. Pink and orange RGs are twins of the red ones.

As already described in Section 3.2, slip deformation in aluminum and copper bicrystals of Type II orientation has common features. However, their recrystallization behaviors are quite different. In the aluminum bicrystals, RGs along the $\mathrm{KBs}$ had $\langle 111\rangle$ rotation relationships with the DM or KBs. $\left.{ }^{15}\right)$ (a)

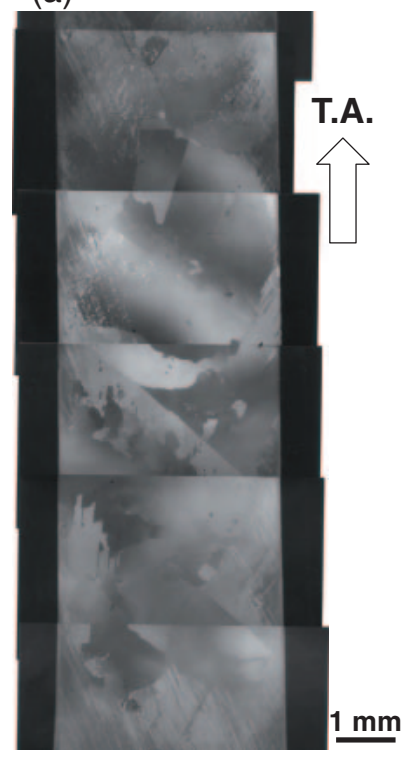

(b)

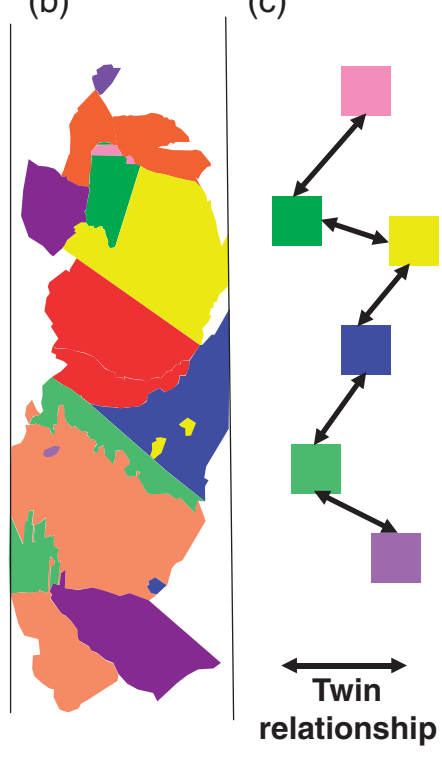

Fig. 8 (a) SEM image of recrystallized Sample A. (b) RGs are colored according to their orientation. (c) Color diagram to show twin relationships between RGs in Sample A.
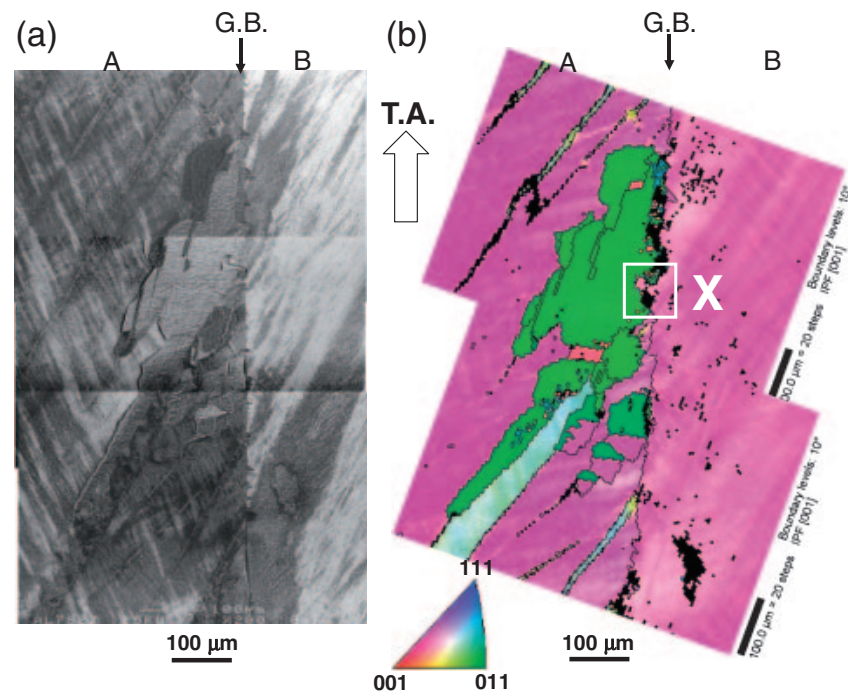

Fig. 9 (a) SEM image of recrystallized region in Sample AB. (b) EBSD orientation map of RGs in Sample AB. The color corresponds to the crystallographic orientation projected onto the tensile axis.

This difference is attributable to the lower stacking fault energy in copper.

In aluminum symmetric bicrystals with a Schmid factor of 0.5 (i.e., Type I bicrystals), annealing induced large-scale SIBM along the KBs. ${ }^{14)}$ On the other hand, in both aluminum ${ }^{15)}$ and copper Type II bicrystals, only small-scale SIBM was found. This comparison suggests the essential conditions for SIBM: an accumulation of edge dislocations at the $\mathrm{DM} / \mathrm{KB}$ boundaries, and a strain energy difference between the KBs in one grain and the DM in the adjacent grain. In Type I bicrystals, the dislocation density in the DM of the adjacent grain is relatively low because only the primary slip system is activated. However, in Type II 


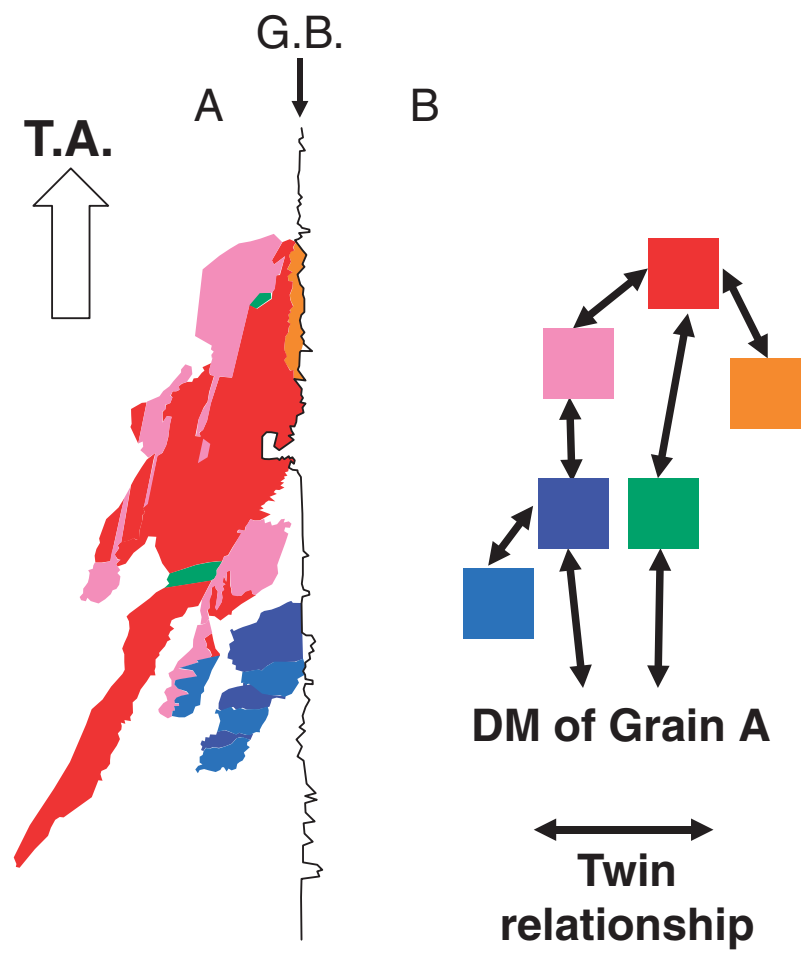

Fig. 10 Color diagram to show twin relationships between DM and RGs in Sample AB.

bicrystals, the activation of the secondary slip system on the conjugate slip plane results in the formation of a double-slip region with high dislocation density in the vicinity of the grain boundary.

\section{Conclusions}

Tensile deformation and subsequent annealing experiments were carried out for copper single crystals and an asymmetric bicrystal with a Schmid factor of 0.5 . Vickers indents were made on one of the single crystals. Results are summarized as follows.

(1) Although the Vickers indents promoted the formation of KBs, the width, slip morphology and crystallographic rotation were the same as for the $\mathrm{KB}$ in the single crystal without indents.

(2) The rotation associated with the KBs in the bicrystal was similar to that in the single crystals irrespective that the width of the KBs was ten times larger.

(3) The density of RGs corresponds well with the development of KBs.

(4) The comparison with aluminum bicrystals of the same orientation made clear that asymmetric deformation about the grain boundary suppressed the large-scale SIBM along the KBs. Recrystallization other than SIBM, i.e., $\langle 111\rangle-$ rotation mechanisms in aluminum and twin-formation in copper, was promoted.

\section{REFERENCES}

1) F. J. Humphreys and M. Hatherly: Recrystallization and Related Annealing Phenomena, (Elsevier, Oxford, 1996) p. 69.

2) H. W. F. Heller, C. A. Verbraak and B. H. Kolster: Acta Metall. 32 (1984) 1395-1406.

3) W. B. Hutchinson: Acta Metall. 37 (1989) 1047-1056.

4) S. Kikuchi, E. Kimura and M. Koiwa: J. Mater. Sci. 27 (1992) 49274934.

5) Y. L. Liu, H. Hu and N. Hansen: Acta Metall. Mater. 43 (1995) 23952405.

6) M. Blicharski, J. Liu and H. Hu: Acta Metall. Mater. 43 (1995) 31253138 .

7) S. Weiß and G. Gottstein: Mater. Sci. Eng. A 256 (1998) 8-17.

8) M. C. Theyssier and J. H. Driver: Mater. Sci. Eng. A 272 (1999) 73-82.

9) K. Kashihara and F. Inoko: Acta Mater. 49 (2001) 3051-3061.

10) J. A. Wert, K. Kashihara, T. Okada, X. Huang and F. Inoko: Philos. Mag. 85 (2005) 1989-2006.

11) K. Higashida, J. Takamura and N. Narita: Mater. Sci. Eng. 81 (1986) 239-258.

12) K. Kashihara, M. Tagami and F. Inoko: Mater. Trans. JIM 37 (1996) 564-571.

13) F. Inoko, M. Kurimoto and K. Kashihara: J. Jpn. Inst. Metals 54 (1990) 642-649.

14) F. Inoko, M. Kobayashi and S. Kawaguchi: Scr. Metall. 21 (1987) 1405-1410.

15) T. Okada, M. Kotaka, T. Wada, M. Tagami and F. Inoko: Mater. Trans. 47 (2006) 838-844.

16) T. Okada, M. Utani, A. Osue, N. Fujii, M. Tagami and F. Inoko: Mater. Trans. 46 (2005) 602-607.

17) J. D. Livingston and B. Chalmers: Acta Metall. 5 (1957) 322-327. 